\title{
A CONVERGENCE ANALYSIS FOR NONSYMMETRIC LANCZOS ALGORITHMS
}

\author{
QIANG YE
}

\begin{abstract}
A convergence analysis for the nonsymmetric Lanczos algorithm is presented. By using a tridiagonal structure of the algorithm, some identities concerning Ritz values and Ritz vectors are established and used to derive approximation bounds. In particular, the analysis implies the classical results for the symmetric Lanczos algorithm.
\end{abstract}

\section{INTRODUCTION}

Lanczos' algorithm is one of the most popular methods for computing some extreme eigenvalues of large symmetric matrices. An elegant theory and analyses of the symmetric Lanczos algorithm have been developed since the 1960's, which include error bounds of Kanial, Paige, and Saad (see [3, 7, 10] or [8]). At the same time, considerable effort has been made to generalize this work to nonsymmetric problems. The idea of tridiagonalization is naturally extended and yields a two-sided nonsymmetric algorithm (see [4, 9, 2]). However, several substantial problems, e.g., a breakdown phenomenon and a convergence analysis, remain unsolved.

The Lanczos algorithm was originally introduced as a method to tridiagonalize a general matrix [4]. Later it was found that it can be used to compute some extreme eigenvalues. It can be regarded, in particular, as a Rayleigh-Ritz projection method using Krylov subspaces; and based on this, a convergence analysis was established using the minimax theorem $[3,7,10]$. Since there is no minimax characterization for general matrices, this analysis cannot easily be generalized. Nevertheless, some approaches have been suggested in this regard. In [11] the idea using projection on Krylov subspaces is extended to show that some eigenvectors are close to the Krylov subspaces. The recent work [1] establishes some properties of the Lanczos polynomials which can be used to explain the convergence of the Lanczos algorithm. In [5, 13], by using a minimax theorem, the classical method is applied to definite matrix pencil problems. However, the result there suggests that the classical approach may not be the best for nonsymmetric problems. We therefore take another look at the Lanczos

Received January 15, 1990; revised May 18, 1990.

1980 Mathematics Subject Classification (1985 Revision). Primary 65F15.

Key words and phrases. Lanczos algorithm, convergence bound, tridiagonal matrices.

Research supported by a University of Calgary Research Fellowship. 
algorithm and find that it is essentially a method of approximating a tridiagonal matrix using its submatrices. It turns out that employing the tridiagonal structure of the algorithm is an appropriate approach.

We shall present a convergence analysis for the nonsymmetric Lanczos algorithms. Our analysis is based on the tridiagonal structure and is completely different from the classical approach. In particular, we shall reprove some classical results, and some of our results are even new for the classical symmetric case.

We first introduce the Lanczos algorithm in $\S 2$. Then some identities are established for tridiagonal matrices in $\S 3$. The approximation bounds are derived for Ritz values in $\S 4$ and for Ritz vectors in $\S 5$. Following that, the implications of this analysis to the symmetric Lanczos algorithm are discussed in $\S 6$. Finally, some numerical examples are presented in $\S 7$ and some remarks in $\S 8$.

Besides the standard notation in numerical analysis, we will use the following notation. $I$ will denote an identity matrix and $I_{m}$ will specify the $m \times m$ identity matrix. $e_{i, m}$ will denote the $i$ th coordinate vector of $R^{m}$, i.e., $I_{m}=$ $\left[e_{1, m}, \ldots, e_{m, m}\right]$.

\section{LANCZOS ALGORITHMS}

In this section, we briefly introduce the Lanczos algorithms. The details can be found in [9] or [2].

Given a matrix $A$ and two starting vectors $x_{1}$ and $y_{1}$ in $C^{n}$, the nonsymmetric Lanczos algorithm, in step $m$, generates sequences $\left\{x_{1}, \ldots, x_{m}\right\}$ and $\left\{y_{1}, \ldots, y_{m}\right\}$ via a three-term recursion, so that

$$
\begin{aligned}
& A Y_{m}-Y_{m} T_{m}=\beta_{m+1} y_{m+1} e_{m, m}^{*}, \\
& X_{m}^{*} A-T_{m} X_{m}^{*}=\gamma_{m+1} e_{m, m} x_{m+1}^{*},
\end{aligned}
$$

and

$$
X_{m}^{*} Y_{m}=I_{m},
$$

where $X_{m}=\left[x_{1}, \ldots, x_{m}\right], Y_{m}=\left[y_{1}, \ldots, y_{m}\right]$, and

$$
T_{m}=\left(\begin{array}{cccc}
\alpha_{1} & \gamma_{2} & & \\
\beta_{2} & \ddots & \ddots & \\
& \ddots & \ddots & \gamma_{m} \\
& & \beta_{m} & \alpha_{m}
\end{array}\right) .
$$

The algorithm continues until breakdown, that is, when $x_{m+1}^{*} y_{m+1}=0$ at some step $m$. This is one of the serious problems in the nonsymmetric Lanczos algorithm (see [9] for a detailed discussion). Numerically, it is rare to have an exact breakdown. The real difficulty comes when the iteration is close to breakdown. In this theoretical analysis, we always assume that no breakdown occurs. 
From the above construction, it is easy to see that

$$
X_{m}^{*} A Y_{m}=T_{m}
$$

and

$$
X_{m}^{*} Y_{m}=I_{m}
$$

In particular, at step $n$, we have $X_{n}^{*}=Y_{n}^{-1}$. Then $A$ is similar to $T_{n}$, and the eigenpairs of $T_{n}$ give those of $A$. This was originally used as a method to tridiagonalize a matrix $A$. However, the attractive feature of the Lanczos algorithm is that it usually stops at $m \ll n$, and some extreme eigenvalues of $T_{m}$ can be used to approximate some eigenvalues of $A$. Specifically, at step $m$, we find the Jordan decomposition of $T_{m}$,

$$
T_{m}=P^{*} \Theta Q, \quad P^{*} Q=I,
$$

where $\Theta$ is the Jordan canonical form of $T_{m}$. Then the eigenvalues of $T_{m}$ (or $\Theta)$ are called Ritz values. Further, letting

$$
U=\left[u_{1}, \ldots, u_{m}\right]=X_{m} Q^{*}, \quad V=\left[v_{1}, \ldots, v_{m}\right]=Y_{m} P^{*},
$$

we call $u_{i}^{*}$ (resp. $v_{i}$ ) a left (resp. right) Ritz vector. We will show that some Ritz values and Ritz vectors give good approximations to the eigenpairs of $A$.

If $A$ is symmetric, we take the initial vectors $x_{1}=y_{1}$. Then the algorithm yields $X_{m}=Y_{m}$ and a symmetric $T_{m}$, which is just the classical symmetric Lanczos algorithm.

\section{TRIDIAgONAL MATRICES}

The symmetric Lanczos algorithm has been successfully treated as a RayleighRitz projection method using Krylov subspaces. For nonsymmetric matrices, it can also be viewed as an oblique projection method (see [11]). The approach is, however, not as successful as in the symmetric case. As mentioned before, the Lanczos algorithm is closely related to its tridiagonal structure. To analyze the algorithm, we first consider tridiagonal matrices.

In the following we always denote an $n \times n$ tridiagonal matrix $T_{n}$ by

$$
T_{n}=\left(\begin{array}{cccc}
\alpha_{1} & \gamma_{2} & & \\
\beta_{2} & \ddots & \ddots & \\
& \ddots & \ddots & \gamma_{n} \\
& & \beta_{n} & \alpha_{n}
\end{array}\right) .
$$

Lemma 3.1. Let $T_{m}$ be a tridiagonal matrix; then $e_{1, m}^{*} T_{m}^{k} e_{m, m}=0$ for $k \leq$ $m-2$ and $e_{1, m}^{*} T_{m}^{m-1} e_{m, m}=\gamma_{2} \cdots \gamma_{m}$.

Proof. It is easy to check that, for $k \leq m-1$,

$$
e_{1, m}^{*} T_{m}^{k}=\left(*, \ldots, *, \gamma_{2} \cdots \gamma_{k+1}, 0, \ldots, 0\right),
$$

where the product of the $\gamma$ 's is in position $k+1$. From this, the lemma follows. 
The next two theorems establish some relations between a tridiagonal matrix and its submatrices.

Theorem 3.2. Let $T_{m}$ be the $m \times m$ leading submatrix of a tridiagonal matrix $T_{n}$. Then

$$
e_{1, n}^{*} T_{n}^{k}=\left(e_{1, m}^{*} T_{m}^{k}, 0\right) \quad \text { for } k \leq m-1
$$

and

$$
T_{n}^{k} e_{1, n}=\left(\begin{array}{c}
T_{m}^{k} e_{1, m} \\
0
\end{array}\right)
$$

Proof. We prove (3.1) only. Let

$$
T_{n}=\left(\begin{array}{cc}
T_{m} & E \\
\widehat{E} & T_{n-m}
\end{array}\right)
$$

where $E=\gamma_{m+1} e_{m, m} e_{1, n-m}^{*}$ and $\widehat{E}=\beta_{m+1} e_{1, n-m} e_{m, m}^{*}$. If, for some $k \leq$ $m-2$,

$$
e_{1, n}^{*} T_{n}^{k}=\left(e_{1, m}^{*} T_{m}^{k}, 0\right),
$$

then

$$
e_{1, n}^{*} T_{n}^{k+1}=\left(e_{1, m}^{*} T_{m}^{k}, 0\right) T_{n}=\left(e_{1, m}^{*} T_{m}^{k+1}, e_{1, m}^{*} T_{m}^{k} E\right)=\left(e_{1, m}^{*} T_{m}^{k+1}, 0\right),
$$

where by Lemma 3.1, $e_{1, m}^{*} T_{m}^{k} E=\gamma_{m+1} e_{1, m}^{*} T_{m}^{k} e_{m, m} e_{1, n-m}^{*}=0$. Hence the theorem follows by induction.

Conceptually, this theorem says that $T_{m}^{k}$ and $T_{n}^{k}$ have essentially the same first row (column) for $k \leq m-1$. Furthermore, $T_{m}^{k}$ and $T_{n}^{k}$ have the same $(1,1)$ element for $k \leq 2 m-1$, as shown in the next theorem.

Theorem 3.3. Let $T_{m}$ be the $m \times m$ leading submatrix of a tridiagonal matrix $T_{n}$. Then

$$
e_{1, n}^{*} T_{n}^{k} e_{1, n}=e_{1, m}^{*} T_{m}^{k} e_{1, m} \text { for } k \leq 2 m-1
$$

and

$$
e_{1, n}^{*} T_{n}^{2 m} e_{1, n}=e_{1, m}^{*} T_{m}^{2 m} e_{1, m}+\beta_{2} \cdots \beta_{m+1} \gamma_{2} \cdots \gamma_{m+1} .
$$

Proof. We first prove by induction that, for $k \geq m+1$,

$$
\begin{aligned}
e_{1, n}^{*} T_{n}^{k}=\left(e_{1, m}^{*} T_{m}^{k}\right. & +e_{1, m}^{*} T_{m}^{m-1} E \hat{E} T_{m}^{k-m-1} \\
& \left.+e_{1, m}^{*} \sum_{i=0}^{k-m-2} G_{i} \hat{E} T_{m}^{i}, e_{1, m}^{*} H\right),
\end{aligned}
$$

where $G_{i}$ and $H$ are $m \times(n-m)$ matrices.

From (3.1), we obtain

$$
e_{1, n}^{*} T_{n}^{m+1}=\left(e_{1, m}^{*} T_{m}^{m+1}+e_{1, m}^{*} T_{m}^{m-1} E \widehat{E}, e_{1, m}^{*} H\right),
$$


where $H=T_{m}^{m} E+T_{m}^{m-1} E T_{n-m}$. We now assume that (3.4) is true for some $k \geq m+1$. Then, for $k+1$,

$$
\begin{aligned}
e_{1, n}^{*} T_{n}^{k+1}=\left(e_{1, m}^{*} T_{m}^{k+1}\right. & +e_{1, m}^{*} T_{m}^{m-1} E \widehat{E} T_{m}^{k-m} \\
& \left.+e_{1, m}^{*} \sum_{i=1}^{k-m-1} G_{i-1} \widehat{E} T_{m}^{i}+e_{1, m}^{*} H \widehat{E}, e_{1, m}^{*} \widehat{H}\right),
\end{aligned}
$$

where $\widehat{H}=T_{m}^{k} E+T_{m}^{m-1} E \widehat{E} T_{m}^{k-m-1} E+\sum_{i=0}^{k-m-2} G_{i} \widehat{E} T_{m}^{i} E+H T_{n-m}$. Letting $\widehat{G}_{i}=G_{i-1}$ and $\widehat{G}_{0}=H$, we obtain (3.4) for $k+1$. So (3.4) is proved.

Now, for $k \leq m$, (3.1) leads to (3.2). For $m+1 \leq k \leq 2 m-1$ and $k=2 m,(3.2)$ and (3.3) follow from (3.4) and Lemma 3.1 by a straightforward computation.

We remark that it is possible to derive some formulae of form (3.3) with larger exponent $k$ by using (3.4). The resulting expression, however, will be very complicated and of little use.

\section{ERROR BOUNDS}

This section will develop error bounds for Ritz values. We define $P^{k}$ to be the set of polynomials of degree not greater than $k$, and $M P^{k}$ to be the set of monic polynomials of degree $k$.

Let the Lanczos algorithm be applied to a matrix $A$ and

$$
A=Z_{r} \Lambda Z_{l}^{*}, \quad Z_{l}^{*} Z_{r}=I,
$$

be the Jordan decomposition of $A$. Then $Z_{r}=\left[z_{1}^{(r)}, \ldots, z_{n}^{(r)}\right]$ (resp. $Z_{l}=$ $\left.\left[z_{1}^{(l)}, \ldots, z_{n}^{(l)}\right]\right)$ contains the right (resp. left) eigenvectors and the generalized eigenvectors. Letting

$$
X=Z_{r}^{*} X_{n}=\left(x_{i j}\right) \text { and } Y=Z_{l}^{*} Y_{n}=\left(y_{i j}\right)
$$

with $X_{n}, Y_{n}$ being generated by $(2.1)$, we have

$$
T_{n}=X^{*} \Lambda Y
$$

and

$$
X^{*} Y=I \text {. }
$$

Note that $x_{i 1}=z_{i}^{(r) *} x_{1}$ is the $z_{i}^{(l)}$ component of the initial vector $x_{1}$, i.e.,

$$
x_{1}=\sum_{i=1}^{n} x_{i 1} z_{i}^{(l)} \text {, }
$$

and $y_{i 1}=z_{i}^{(l) *} y_{1}$ is the $z_{i}^{(r)}$ component of the initial vector $y_{1}$, i.e.,

$$
y_{1}=\sum_{i=1}^{n} y_{i 1} z_{i}^{(r)} \text {. }
$$

Using the properties obtained in $\S 3$, we establish the first theorem. 
Theorem 4.1. Let $X=\left(x_{i j}\right), Y=\left(y_{i j}\right)$ and $P=\left(p_{i j}\right), Q=\left(q_{i j}\right)$ be defined as in (4.1) and (2.2), respectively, and let

$$
\Lambda=\operatorname{diag}\left[\Lambda_{1}, \lambda_{s+1}, \ldots, \lambda_{n}\right], \quad \Theta=\operatorname{diag}\left[\Theta_{1}, \theta_{t+1}, \ldots, \theta_{m}\right],
$$

where

$$
\Lambda_{1}=\left(\begin{array}{cccc}
\lambda_{1} & 1 & & \\
& \ddots & \ddots & \\
& & \ddots & 1 \\
& & & \lambda_{1}
\end{array}\right) \in C^{s \times s}, \quad \Theta_{1}=\left(\begin{array}{cccc}
\theta_{1} & 1 & & \\
& \ddots & \ddots & \\
& & \ddots & 1 \\
& & & \theta_{1}
\end{array}\right) \in C^{t \times t} .
$$

Then, for any $f \in P^{2 m-1}$,

$$
\sum_{i=0}^{s-1} f^{(i)}\left(\lambda_{1}\right) \omega_{i}+\sum_{i=s+1}^{n} f\left(\lambda_{i}\right) \bar{x}_{i 1} y_{i 1}=\sum_{i=0}^{t-1} f^{(i)}\left(\theta_{1}\right) \hat{\omega}_{i}+\sum_{i=t+1}^{m} f\left(\theta_{i}\right) \bar{p}_{i 1} q_{i 1},
$$

and, for any $f \in M P^{2 m}$,

$$
\begin{aligned}
& \sum_{i=0}^{s-1} f^{(i)}\left(\lambda_{1}\right) \omega_{i}+\sum_{i=s+1}^{n} f\left(\lambda_{i}\right) \bar{x}_{i 1} y_{i 1} \\
& =\sum_{i=0}^{t-1} f^{(i)}\left(\theta_{1}\right) \hat{\omega}_{i}+\sum_{i=t+1}^{m} f\left(\theta_{i}\right) \bar{p}_{i 1} q_{i 1}+\prod_{j=2}^{m+1} \beta_{j} \gamma_{j},
\end{aligned}
$$

where $\omega_{i}=\frac{1}{i !} \sum_{j=1}^{s-i} \bar{x}_{j 1} y_{(j+i), 1}$ and $\hat{\omega}_{i}=\frac{1}{i !} \sum_{j=1}^{t-i} \bar{p}_{j 1} q_{(j+i), 1}$.

Proof. Substituting (4.2) and (2.2) into (3.2) of Theorem 3.3, we obtain

$$
e_{1, n}^{*} X^{*} \Lambda^{k} Y e_{1, n}=e_{1, m}^{*} P^{*} \Theta^{k} Q e_{1, m}
$$

for $k \leq 2 m-1$. Then, for any $f \in P^{2 m-1}$, we have

$$
e_{1, n}^{*} X^{*} f(\Lambda) Y e_{1, n}=e_{1, m}^{*} P^{*} f(\Theta) Q e_{1, m} .
$$

A straightforward computation from this proves (4.4). Similarly, we can prove (4.5), using (3.3).

We can use this theorem to derive some identities concerning approximation errors. We state the following theorem for a diagonalizable matrix.

Theorem 4.2. Let $A$ be a matrix with $n$ distinct eigenvalues and $\left|\lambda_{1}-\theta_{k}\right|=$ $\min _{j}\left|\lambda_{1}-\theta_{j}\right|$.

(1) If $k \geq 2$, i.e., $\theta_{k}$ is a semisimple eigenvalue, then, for any $h \in P^{2 m-2}$,

$$
\begin{aligned}
\lambda_{1}-\theta_{k}=\frac{1}{h\left(\lambda_{1}\right) \bar{x}_{11} y_{11}}( & -\sum_{i=2}^{n}\left(\lambda_{i}-\theta_{k}\right) h\left(\lambda_{i}\right) \bar{x}_{i 1} y_{i 1} \\
& \left.+\sum_{i=0}^{t-1} h^{(i)}\left(\theta_{i}\right) \sigma_{i}+\sum_{i=t+1}^{m}\left(\theta_{i}-\theta_{k}\right) h\left(\theta_{i}\right) \bar{p}_{i 1} q_{i 1}\right),
\end{aligned}
$$

where $\sigma_{i}=\left(\theta_{1}-\theta_{k}\right) \hat{\omega}_{i}+(i+1) \hat{\omega}_{i+1}$ with $\hat{\omega}_{t}=0$. 
(2) If $k=1$, then, for any $h \in P^{2 m-t-1}$,

$$
\begin{aligned}
\left(\lambda_{1}-\theta_{1}\right)^{t}=\frac{1}{h\left(\lambda_{1}\right) \bar{x}_{11} y_{11}} & -\sum_{i=2}^{n}\left(\lambda_{i}-\theta_{1}\right)^{t} h\left(\lambda_{i}\right) \bar{x}_{i 1} y_{i 1} \\
& \left.+\sum_{i=t+1}^{m}\left(\theta_{i}-\theta_{1}\right)^{t} h\left(\theta_{i}\right) \bar{p}_{i 1} q_{i 1}\right) .
\end{aligned}
$$

Proof. (1) For $k \geq 2$, we substitute $f(x)=\left(x-\theta_{k}\right) h(x)$ into (4.4) and obtain

$$
\begin{aligned}
\left(\lambda_{1}-\right. & \left.\theta_{k}\right) h\left(\lambda_{1}\right) \bar{x}_{11} y_{11}+\sum_{i=2}^{n}\left(\lambda_{i}-\theta_{k}\right) h\left(\lambda_{i}\right) \bar{x}_{i 1} y_{i 1} \\
= & \sum_{i=0}^{t-1} f^{(i)}\left(\theta_{1}\right) \hat{\omega}_{i}+\sum_{i=t+1}^{m}\left(\theta_{i}-\theta_{k}\right) h\left(\theta_{i}\right) \bar{p}_{i 1} q_{i 1} .
\end{aligned}
$$

It is easy to check that

$$
\sum_{i=0}^{t-1} f^{(i)}\left(\theta_{i}\right) \hat{\omega}_{i}=\sum_{i=0}^{t-1} h^{(i)}\left(\theta_{i}\right) \sigma_{i}
$$

This leads to (4.6).

(2) For $k=1$, we substitute $f(x)=\left(x-\theta_{1}\right)^{t} h(x)$ into (4.4). Since $f^{(i)}\left(\theta_{1}\right)=$ $0,1 \leq i \leq t-1$, we get

$$
\left(\lambda_{1}-\theta_{1}\right)^{t} h\left(\lambda_{1}\right) \bar{x}_{11} y_{11}+\sum_{i=2}^{n}\left(\lambda_{i}-\theta_{1}\right)^{t} h\left(\lambda_{i}\right) \bar{x}_{i 1} y_{i 1}=\sum_{i=t+1}^{m}\left(\theta_{i}-\theta_{1}\right)^{t} h\left(\theta_{i}\right) \bar{p}_{i 1} q_{i 1} .
$$

This leads to (4.7).

Obviously, our method is not restricted to diagonalizable matrices. Instead, it applies to any eigenvalues of a general matrix. For instance, if $\lambda_{1}$ is an eigenvalue with Jordan block of size $s$, we can use $f(x)=\left(x-\lambda_{1}\right)^{s} h(x)$ in (4.4) and subsequently obtain similar identities. However, the results are more complicated. Such statements are therefore simply omitted.

To use the theorem, we choose for $h$ a polynomial $p$ so that $p\left(\lambda_{1}\right)=1$ and $p\left(\lambda_{i}\right) \quad(i \neq 1), p\left(\theta_{i}\right) \quad(i \neq k)$ are as small as possible. Then the right-hand side of (4.6) or (4.7) is a small number, and $\lambda_{1}-\theta_{k}$ can be bounded by this number. Clearly, the magnitude of the bound depends on the distribution of $\lambda_{i}, \theta_{i}$. On the other hand, comparison between (4.6) and (4.7) suggests that convergence of a Ritz value with Jordan block of size $s$ is expected to slow down by an order of $s$.

To present some detailed bounds, we will concentrate on the case where both $A$ and $T_{m}$ are diagonalizable. Let $\sigma(A)=\left\{\lambda_{1}, \ldots, \lambda_{n}\right\}$ and $\sigma\left(T_{m}\right)=$ $\left\{\theta_{1}, \ldots, \theta_{m}\right\}$ be the spectra of $A$ and $T_{m}$, respectively, and let

$$
\sigma_{1}=\left\{\lambda_{2}, \ldots, \lambda_{n}\right\}, \quad \hat{\sigma}_{1}=\left\{\theta_{2}, \ldots, \theta_{m}\right\}
$$


We then define

$$
\varepsilon_{1}^{(k)}(S)=\inf _{p \in P^{k}, p\left(\lambda_{1}\right)=1} \max _{x \in S}|p(x)|
$$

and

$$
\delta_{1}(S)=\max \left\{\left|x-\theta_{1}\right| \prod_{\lambda \in S} \frac{|x-\lambda|}{\left|\lambda_{1}-\lambda\right|}: x \in \sigma_{1} \cup \hat{\sigma}_{1}\right\}
$$

for $S \subset \sigma_{1} \cup \hat{\sigma}_{1}$.

Corollary 4.3. Let $A$ and $T_{m}$ be diagonalizable, with $\sigma(A)=\left\{\lambda_{1}, \ldots, \lambda_{n}\right\}$ and $\sigma\left(T_{m}\right)=\left\{\theta_{1}, \ldots, \theta_{m}\right\}$. Assume that $\left|\lambda_{1}-\theta_{1}\right|=\min _{j}\left|\lambda_{1}-\theta_{j}\right|$, and let $\sigma_{1}=\left\{\lambda_{2}, \ldots, \lambda_{n}\right\}, \hat{\sigma}_{1}=\left\{\theta_{2}, \ldots, \theta_{m}\right\}$.

(1) If $\sigma_{1} \cup \hat{\sigma}_{1}=S_{1} \cup S_{2}$ with $S_{1}$ and $S_{2}$ disjoint, and $s=\left|S_{2}\right| \leq 2 m-2$, then

$$
\left|\lambda_{1}-\theta_{1}\right| \leq \varepsilon_{1}^{(2 m-2-s)}\left(S_{1}\right) \delta_{1}\left(S_{2}\right)
$$

$$
\times \frac{\left(\sum_{i=2}^{n}\left|x_{i 1}\right|^{2}+\sum_{i=2}^{m}\left|p_{i 1}\right|^{2}\right)^{1 / 2}}{\left|x_{11}\right|} \frac{\left(\sum_{i=2}^{n}\left|y_{i 1}\right|^{2}+\sum_{i=2}^{m}\left|q_{i 1}\right|^{2}\right)^{1 / 2}}{\left|y_{11}\right|} .
$$

(2) If $\sigma_{1}=S_{1} \cup S_{2}$ with $S_{1}$ and $S_{2}$ disjoint, and $s=\left|S_{2}\right| \leq m-1$, then

$$
\left|\lambda_{1}-\theta_{1}\right| \leq \varepsilon_{1}^{(m-1-s)}\left(S_{1}\right) \delta_{1}\left(S_{2} \cup \hat{\sigma}_{1}\right) \frac{\left(\sum_{i=2}^{n}\left|x_{i 1}\right|^{2}\right)^{1 / 2}}{\left|x_{11}\right|} \frac{\left(\sum_{i=2}^{n}\left|y_{i 1}\right|^{2}\right)^{1 / 2}}{\left|y_{11}\right|} .
$$

Proof. (1) Substituting $h(x)=p(x) \prod_{\lambda \in S_{2}}(x-\lambda)$ for any $p \in P^{2 m-2-s}$ with $p\left(\lambda_{1}\right)=1$ into (4.6), we obtain

$$
\begin{aligned}
&\left|\lambda_{1}-\theta_{1}\right|=\frac{1}{\left|x_{11} y_{11}\right|} \mid-\sum_{\lambda_{i} \in S_{1}}\left(\lambda_{i}-\theta_{1}\right) p\left(\lambda_{i}\right) \bar{x}_{i 1} y_{i 1} \prod_{\lambda \in S_{2}} \frac{\left(\lambda_{i}-\lambda\right)}{\left(\lambda_{1}-\lambda\right)} \\
&+\sum_{\theta_{i} \in S_{1}}\left(\theta_{i}-\theta_{1}\right) p\left(\theta_{i}\right) \bar{p}_{i 1} q_{i 1} \prod_{\lambda \in S_{2}} \frac{\left(\theta_{i}-\lambda\right)}{\left(\lambda_{1}-\lambda\right) \mid} \\
& \leq \max _{x \in S_{1}}|p(x)| \delta_{1}\left(S_{2}\right)\left(\sum_{\lambda_{i} \in S_{2}}\left|x_{i 1} y_{i 1}\right|+\sum_{\theta_{i} \in S_{2}}\left|p_{i 1} q_{i 1}\right|\right) /\left|x_{11} y_{11}\right| \\
& \leq \max _{x \in S_{1}}|p(x)| \delta_{1}\left(S_{2}\right) \frac{\left(\sum_{i=2}^{n}\left|x_{i 1}\right|^{2}+\sum_{i=2}^{m}\left|p_{i 1}\right|^{2}\right)^{1 / 2}}{\left|x_{11}\right|} \\
& \times \frac{\left(\sum_{i=2}^{n}\left|y_{i 1}\right|^{2}+\sum_{i=2}^{m}\left|q_{i 1}\right|^{2}\right)^{1 / 2}}{\left|y_{11}\right|} .
\end{aligned}
$$

This proves (4.8). 
(2) Substituting $h(x)=p(x) \prod_{\lambda \in S_{2} \cup \hat{\sigma}_{1}}(x-\lambda)$ for any $p \in P^{m-1-s}$ with $p\left(\lambda_{1}\right)=1$ into (4.6), we obtain

$$
\begin{aligned}
\left|\lambda_{1}-\theta_{1}\right| & =\frac{1}{\left|x_{11} y_{11}\right|}\left|\sum_{\lambda_{i} \in S_{1}}\left(\lambda_{i}-\theta_{1}\right) p\left(\lambda_{i}\right) \bar{x}_{i 1} y_{i 1} \prod_{\lambda \in S_{2} \cup \hat{\sigma}_{1}} \frac{\left(\lambda_{i}-\lambda\right)}{\left(\lambda_{1}-\lambda\right)}\right| \\
& \leq \max _{x \in S_{1}}|p(x)| \delta_{1}\left(S_{2} \cup \hat{\sigma}_{1}\right) \frac{\left(\sum_{i=2}^{n}\left|x_{i 1}\right|^{2}\right)^{1 / 2}}{\left|x_{11}\right|} \frac{\left(\sum_{i=2}^{n}\left|y_{i 1}\right|^{2}\right)^{1 / 2}}{\left|y_{11}\right|} .
\end{aligned}
$$

This proves (4.9).

We now analyze the magnitude of $\varepsilon_{1}^{(k)}\left(S_{1}\right)$. In [11], it is proved that if $k<\left|S_{1}\right|$, then there exists $\left\{\alpha_{1}, \ldots, \alpha_{k+1}\right\} \subset S_{1}$ such that

$$
\varepsilon_{1}^{(k)}\left(S_{1}\right)=\left(\sum_{j=1}^{k+1} \prod_{i \neq j} \frac{\left|\alpha_{i}-\lambda_{1}\right|}{\left|\alpha_{i}-\lambda_{j}\right|}\right)^{-1}
$$

Furthermore, it is shown from this that $\varepsilon_{1}^{(k)}\left(S_{1}\right)$ is small when $\lambda_{1}$ is well separated from $\left\{\alpha_{1}, \ldots, \alpha_{k+1}\right\}$. For details see [11]. Another analysis can be conducted using Chebyshev polynomials $[11,6]$. Let $S_{1}$ lie inside an ellipse and $\lambda_{1}$ lie outside of it and on the major axis. More specifically, by a shift and rotation we can assume that $\lambda_{1}=0$ and $S_{1}$ lies inside of the ellipse $E$ which is centered at $d$ and has foci at $d+c$ and $d-c$ and semimajor axis $a$ with $0<c \leq a \leq d$ (i.e., the real axis is the major axis of $E$ and the origin lies outside of $E$ ). Let $T_{k}(x)$ denote the Chebyshev polynomial of degree $k$ on the interval $[-1,1]$ (see [6] or [8] for a detailed definition). Then

$$
\min _{p \in P^{k}, p\left(\lambda_{1}\right)=1} \max _{x \in E}|p(x)|=\max _{x \in E}\left|p_{k}(x)\right|=T_{k}\left(\frac{a}{c}\right) / T_{k}\left(\frac{d}{c}\right),
$$

where $p_{k}(x)=T_{k}\left(\frac{d-x}{c}\right) / T_{k}\left(\frac{d}{c}\right)$ (see [6] and references therein). Hence,

$$
\varepsilon_{1}^{(k)}\left(S_{1}\right) \leq T_{k}\left(\frac{a}{c}\right) / T_{k}\left(\frac{d}{c}\right) .
$$

Since $1 \leq \frac{a}{c} \leq \frac{d}{c}$, we have $T_{k}\left(\frac{a}{c}\right) \leq T_{k}\left(\frac{d}{c}\right)$. Furthermore, the bigger the difference between $\frac{a}{c}$ and $\frac{d}{c}$, the smaller is the bound of (4.10). Note that $\frac{d}{c}$ is a measure of separation of $\lambda_{1}$ from $E$, and $\frac{a}{c}$ is a measure of flatness of the ellipse $E$.

On the other hand, if $s=\left|S_{2}\right|$ is small, $\delta_{1}\left(S_{2}\right)$ is a bounded number. If $s=\left|S_{2}\right|$ is large and, in addition, $|x-\lambda|<\left|\lambda_{i}-\lambda\right|$ for most $\lambda \in S_{2}$ and any $x \in S_{1}$, then $\delta_{1}\left(S_{2}\right)$ is a product of $s$ numbers, most of which are less than one. Hence it is a small number.

Thus, for an extreme eigenvalue $\lambda_{1}$, we can partition $\sigma_{1} \cup \hat{\sigma}_{1}$ into a union of $S_{1}$ and $S_{2}$, so that $S_{1}$ lies in a flat ellipse well separated from $\lambda_{1}$ and $t=\left|S_{2}\right|$ is small. Then Corollary 4.3 says that we can expect a good approximation bound for $\lambda_{1}$. 
An alternative partition is by taking $S_{1}$ and $S_{2} \subset \sigma_{1} \cup \hat{\sigma}_{1}$ in (4.8) (or $S_{2} \subset \hat{\sigma}_{1}$ in (4.9), respectively), with $\left|S_{2}\right|=2 m-2$ (resp. $\left|S_{2}\right|=m-1$ ). Since $\varepsilon_{1}^{(0)}\left(S_{1}\right)=$ 1 , we have the following

Corollary 4.4. Under the hypotheses of Corollary 4.3, we have

$$
\begin{aligned}
\left|\lambda_{1}-\theta_{1}\right| \leq \min _{S \subset \sigma_{1} \cup \hat{\sigma}_{1},|S|=2 m-2} \delta_{1}(S) & \frac{\left(\sum_{i=2}^{n}\left|x_{i 1}\right|^{2}+\sum_{i=2}^{m}\left|p_{i 1}\right|^{2}\right)^{1 / 2}}{\left|x_{11}\right|} \\
& \times \frac{\left(\sum_{i=2}^{n}\left|y_{i 1}\right|^{2}+\sum_{i=2}^{m}\left|q_{i 1}\right|^{2}\right)^{1 / 2}}{\left|y_{11}\right|}
\end{aligned}
$$

and

$$
\left|\lambda_{1}-\theta_{1}\right| \leq \min _{S \subset \sigma_{1},|S|=m-1} \delta_{1}\left(S \cup \hat{\sigma}_{1}\right) \frac{\left(\sum_{i=2}^{n}\left|x_{i 1}\right|^{2}\right)^{1 / 2}}{\left|x_{11}\right|} \frac{\left(\sum_{i=2}^{n}\left|y_{i 1}\right|^{2}\right)^{1 / 2}}{\left|y_{11}\right|}
$$

Finally, we remark that analogues of Theorem 4.2 and Corollaries 4.3 and 4.4 can be obtained by using (4.5) with polynomials of higher degree. However, this increase in degree is not significant, and all such statements are therefore omitted.

\section{RITZ VECTORS}

In the previous section, we only considered the convergence of Ritz values. We note that the behavior of Ritz vectors could be quite different from that of Ritz values. In some cases it is more appropriate to consider Ritz vectors and invariant subspaces, e.g., when there are some close eigenvalues, or eigenvalues with Jordan block of size greater than two.

In this section, we give an analysis for Ritz vectors. Again, we use the properties of tridiagonal matrices developed in $\S 3$. For simplicity, we always discuss the case where both $A$ and $T_{m}$ are diagonalizable.

Theorem 5.1. Assume that $A$ and $T_{m}$ are diagonalizable. Then, for any $f \in$ $P^{m-1}$,

$$
\sum_{i=1}^{n} z_{i}^{(r)} f\left(\lambda_{i}\right) y_{i 1}=\sum_{i=1}^{m} v_{i} f\left(\theta_{i}\right) q_{i 1}
$$

where $z_{i}^{(r)}$ are the right eigenvectors and $v_{i}$ the right Ritz vectors.

Proof. By Theorem 3.1, for any $f \in P^{m-1}$,

$$
f\left(T_{n}\right) e_{1, n}=\left(\begin{array}{c}
f\left(T_{m}\right) e_{1, m} \\
0
\end{array}\right) \text {. }
$$

By (4.2) and (2.2), we have

$$
X^{*} f(\Lambda) Y e_{1, n}=\left(\begin{array}{c}
P^{*} f(\boldsymbol{\Theta}) Q e_{1, m} \\
0
\end{array}\right)
$$


or

$$
Z_{r} f(\Lambda) Y e_{1, n}=Y_{n}\left(\begin{array}{c}
P^{*} f(\boldsymbol{\Theta}) Q e_{1, m} \\
0
\end{array}\right)=V f(\boldsymbol{\Theta}) Q e_{1, m} .
$$

Expanding this, we obtain (5.1).

For some $\lambda_{k}$ and $\theta_{l}$, which are close to each other, we choose $f$ so that $f\left(\lambda_{i}\right) \quad(i \neq k)$ and $f\left(\theta_{i}\right) \quad(i \neq l)$ are small and $f\left(\lambda_{k}\right)=1$. Assume that $f\left(\theta_{l}\right) q_{l 1} \neq 0$. Then

where $\alpha$ is a constant and

$$
v_{l}=\alpha\left(z_{k}^{(r)}+w_{\varepsilon}\right)
$$

$$
w_{\varepsilon}=\sum_{i \neq k} z_{i}^{(r)} f\left(\lambda_{i}\right) \frac{y_{i 1}}{y_{k 1}}-\sum_{i \neq l} v_{i} f\left(\theta_{i}\right) \frac{q_{i 1}}{y_{k 1}}
$$

is a small vector compared to $z_{k}^{(r)}$. A particular choice of $g_{l}(x)=$ $\left(x-\theta_{1}\right) \cdots\left(x-\theta_{l-1}\right)\left(x-\theta_{l+1}\right) \cdots\left(x-\theta_{m}\right)$ yields an expression of $v_{l}$ in terms of $z_{k}^{(r)}$.

Corollary 5.2. Let $g_{l}(x)=\left(x-\theta_{1}\right) \cdots\left(x-\theta_{l-1}\right)\left(x-\theta_{l+1}\right) \cdots\left(x-\theta_{m}\right)$. Under the hypotheses of Theorem 5.1 and $q_{l 1} \neq 0$, we have

$$
v_{l}=\alpha \sum_{i=1}^{n} z_{i}^{(r)} g_{l}\left(\lambda_{i}\right) y_{i 1}
$$

for some constant $\alpha$.

For a fixed $l$, there are some $\lambda_{i}$ close to $\theta_{j}(j \neq l)$, in which case $g_{l}\left(\lambda_{i}\right)$ is relatively small. Hence, $v_{l}$ is close to some spectral subspace, though $\theta_{l}$ may not be close to any eigenvalue. So in this case, $v_{l}$ can make a good starting vector to find the remaining eigenvalues.

\section{THE SYMMETRIC CASE}

In this section we apply our techniques to the classical symmetric case. In particular, we are going to derive some generalizations of the classical bound.

For a symmetric matrix $A$, all the eigenvalues $\lambda_{i}$ and the Ritz values are real. Let $\lambda_{1} \leq \cdots \leq \lambda_{n}$ and $\theta_{1} \leq \cdots \leq \theta_{m}$. Then $\lambda_{i} \leq \theta_{i}$ for $i=1, \ldots, m$. The classical convergence analysis compares $\lambda_{i}$ with $\theta_{i}$, which is not necessarily the best approximation to $\lambda_{i}$. For example, if the initial vector $x_{1}$ has a significantly small component in the direction of the eigenvector associated with $\lambda_{1}$, then $\theta_{1}$ will converge to $\lambda_{2}$ first. In such a case, a bound on $\left|\lambda_{1}-\theta_{1}\right|$ is irrelevant. Without using the minimax theorem, our method does not require this match in ordering. This allows us to compare an eigenvalue with the Ritz value that is closest to it.

When considering the left end of the eigenvalues, we note that $\theta_{i}$ decreases as $m$ increases. Then an approximation of $\theta_{l}$ to $\lambda_{k}$ can only be improved if $\theta_{l}>\lambda_{k}$. Otherwise, $\theta_{l}$ will depart from $\lambda_{k}$ and approach $\lambda_{k-1}$. From this point of view, we naturally consider $\lambda_{k}$ approximated by some $\theta_{l}>\lambda_{k}$.

We have seen in $\S 2$ that the symmetric Lanczos algorithm can be obtained by taking $x_{1}=y_{1}$ in the nonsymmetric case. Furthermore, we have $X_{n}=Y_{n}$, 
$X=Y$, and $P=Q$. Combining this with the previous results, we obtain the following

Theorem 6.1. For a fixed $\lambda_{k}$, let $\theta_{l-1}-\lambda_{k}<0<\theta_{l}-\lambda_{k}$ (where $\left.\theta_{0}=-\infty\right)$. Then

$$
\left|\lambda_{k}-\theta_{l}\right| \leq\left|\lambda_{n}-\lambda_{k}\right| \frac{\sum_{i=k+1}^{n}\left|x_{i 1}\right|^{2}}{\left|x_{k 1}\right|^{2}} \inf \left\{\max _{k+1 \leq i \leq n}\left|h\left(\lambda_{i}\right)\right|: h \in \Phi\right\},
$$

where $\Phi=\left\{h \in P^{2(m-1)}: h\left(\lambda_{k}\right)=1, h\left(\theta_{i}\right) \leq 0(1 \leq i \leq l-1)\right.$ and $h\left(\theta_{i}\right) \geq 0$ $(l+1 \leq i \leq m)\}$.

Proof. By Theorem 4.2, for $h \in \Phi$, we have

$$
\begin{aligned}
0 & \leq-\lambda_{k}+\theta_{l}=\frac{\sum_{i \neq k}\left(\lambda_{i}-\theta_{l}\right) h\left(\lambda_{i}\right)\left|x_{i 1}\right|^{2}-\sum_{i \neq l}\left(\theta_{i}-\theta_{l}\right) h\left(\theta_{i}\right)\left|p_{i 1}\right|^{2}}{h\left(\lambda_{k}\right)\left|x_{i 1}\right|^{2}} \\
& \leq \frac{\sum_{i=k+1}^{n}\left(\lambda_{i}-\theta_{l}\right) h\left(\lambda_{i}\right)\left|x_{i 1}\right|^{2}}{h\left(\lambda_{k}\right)\left|x_{i 1}\right|^{2}} \\
& \leq\left|\lambda_{n}-\lambda_{k}\right| \frac{\sum_{i=k+1}^{n}\left|x_{i 1}\right|^{2}}{\left|x_{k 1}\right|^{2}} \max _{k+1 \leq i \leq n}\left|h\left(\lambda_{i}\right)\right| .
\end{aligned}
$$

This proves the theorem.

Now consider the polynomial

$$
h(x)=\left(x-\theta_{1}\right)^{2} \cdots\left(x-\theta_{l-1}\right)^{2} T_{m-l}^{2}\left(\frac{2 x-\lambda_{k+1}-\lambda_{n}}{\lambda_{n}-\lambda_{k+1}}\right) .
$$

It is easy to see that $h(x) / h\left(\lambda_{k}\right) \in \Phi$. Hence, we obtain the following more general form of the classical bound (see [8]).

Corollary 6.2. Under the hypotheses of Theorem 6.1,

$$
\left|\lambda_{k}-\theta_{l}\right| \leq\left|\lambda_{n}-\lambda_{k}\right| \frac{\sum_{i=k+1}^{n}\left|x_{i 1}\right|^{2}}{\left|x_{k 1}\right|^{2}} \prod_{i=1}^{l-1} \frac{\left(\lambda_{n}-\theta_{i}\right)^{2}}{\left(\lambda_{k}-\theta_{i}\right)^{2}} / T_{m-l}^{2}\left(\frac{2 \lambda_{k}-\lambda_{k+1}-\lambda_{n}}{\lambda_{n}-\lambda_{k+1}}\right) .
$$

For Ritz vectors, Theorem 5.1 and Corollary 5.2 apply and give two new results. In particular, we notice that $\sum_{i=1}^{m} v_{i} f\left(\theta_{i}\right) q_{i 1}$ lies in the Krylov subspace. Taking

$$
h(x)=\left(x-\theta_{1}\right) \cdots\left(x-\theta_{l-1}\right) T_{m-l}\left(\frac{2 x-\lambda_{k+1}-\lambda_{n}}{\lambda_{n}-\lambda_{k+1}}\right)
$$

in Theorem 5.1, we can obtain the bound of Saad concerning eigenvectors. We will not state this result, but refer to [10] or [8] for the details. 


\section{EXAMPLeS}

We present two simple examples in this section. Both are taken from the nonsymmetric examples of [1]. For the sake of computational convenience, we have used bound (4.11) in our calculation.

Example 1. Let the matrix be

$$
\begin{aligned}
A & =\operatorname{diag}[70-70 i,-40+80 i, 8-7 i,-1-5 i, 8] \\
& =\operatorname{diag}\left[\lambda_{1}, \lambda_{2}, \lambda_{3}, \lambda_{4}, \lambda_{5}\right],
\end{aligned}
$$

and the initial vectors be $x_{1}=y_{1}=[1,1,1,1,1]^{*}$. Then for $m=3$, the three Ritz values are

$$
\theta_{1}=69.9-70.0 i, \quad \theta_{2}=-40.0+80.1 i, \quad \theta_{3}=4.9-4.0 i
$$

to one decimal digit of accuracy. By taking $S=\left\{\lambda_{2}, \lambda_{5}\right\}$ in (4.11), we obtain the bound 0.346 for $\lambda_{1}-\theta_{1}$. By taking $S=\left\{\lambda_{1}, \lambda_{5}\right\}$, we obtain the bound 0.348 for $\lambda_{2}-\theta_{2}$.

Example 2. In this example,

$$
\begin{aligned}
A & =U \operatorname{diag}[10,13-4 i, 7+3 i,-80 i,-20+90 i] U^{-1} \\
& =U \operatorname{diag}\left[\lambda_{5}, \lambda_{4}, \lambda_{3}, \lambda_{2}, \lambda_{1}\right] U^{-1},
\end{aligned}
$$

with $U$ and the initial vectors chosen randomly as

$$
U=\left(\begin{array}{rrrrr}
2113+2922 i & 6284+5015 i & 5608+9185 i & 2321+2860 i & 3076+6857 i \\
7560+5664 i & 8497+4369 i & 6624+0437 i & 2312+1280 i & 9330+1531 i \\
2+4826 i & 6857+2693 i & 7264+4819 i & 2165+7783 i & 2146+6971 i \\
3303+3322 i & 8782+6326 i & 1985+2640 i & 8834+2119 i & 3126+8416 i \\
6654+5935 i & 684+4052 i & 5443+4148 i & 6525+1121 i & 3616+4062 i
\end{array}\right)
$$

and

$$
x_{1}=y_{1}=\left(\begin{array}{c}
1.0942+0.2736 i \\
0.6524-0.1925 i \\
0.6630-0.7264 i \\
0.4387+0.5967 i \\
0.0888+0.5170 i
\end{array}\right)
$$

For $m=3$, the three Ritz values are

$\theta_{1}=-19.9524+90.2763 i, \quad \theta_{2}=0.1894-79.8844 i, \quad \theta_{3}=11.6666+0.9774 i$.

By taking $S=\left\{\lambda_{2}, \lambda_{4}\right\}$ in (4.11), the bound for $\lambda_{1}-\theta_{1}$ is 0.435 . By taking $S=\left\{\lambda_{1}, \lambda_{3}\right\}$ the bound for $\lambda_{2}-\theta_{2}$ is 1.62 .

\section{ConClusion}

The analysis of the nonsymmetric Lanczos algorithm is considerably more complicated than that of the symmetric one. In this paper, we have developed a convergence analysis which leads to the classical results for symmetric matrices. To our knowledge, it is the only analysis of this kind. Furthermore, the analysis 
of Ritz vectors is new and demonstrates that some Ritz vectors which do not give good approximations of eigenvectors may still be close to some small spectral subspaces.

We remark that all the approximation bounds derived in this paper are not intended to provide a practical computable estimation of the number of iterations needed, but rather to demonstrate the convergence of the Lanczos algorithm.

As is known, the error bound for the largest or smallest eigenvalue in the symmetric Lanczos algorithm depends only on the matrix $A$ and the initial vector $x_{1}$. In contrast to this, the bounds for all eigenvalues in the nonsymmetric Lanczos algorithm depend on Ritz values as well. Unfortunately, there is no guarantee that Ritz values will be well distributed. Indeed for nonsymmetric matrices, the Ritz values can be anywhere in $C$. Then there could be no convergence at all. At this point we should mention that when we talk about convergence of the Lanczos algorithms, it is not strictly in the sense of mathematical convergence, even for the symmetric Lanczos algorithm. As is shown in [12], there are always contrived choices of initial vectors that give rise to nonconvergence of the Lanczos process. From this point of view, it is not reasonable to expect a bound that guarantees convergence all the time, but only a bound that reveals the convergence behavior. Of course, a bound depending only on the matrix and the initial vectors would be most desirable.

\section{ACKNOWLEDGMENT}

I would like to thank Dr. P. Lancaster for his interest and comments. I would also like to thank Dr. Y. Huang for his help.

\section{BIBLIOGRAPHY}

1. G. Cybenko, An explicit formula for Lanczos polynomials, Linear Algebra Appl. 88/89 (1987), 99-115.

2. G. H. Golub and C. F. Van Loan, Matrix computations, The John Hopkins University Press, Baltimore, 1983.

3. S. Kaniel, Estimate for some computational techniques in linear algebra, Math. Comp. 20 (1966), 369-378.

4. C. Lanczos, An iteration method for the solution of the eigenvalue problem of linear differential and integral operators, J. Res. Nat. Bur. Standards Sect. B 45 (1950), 225-280.

5. P. Lancaster and Q. Ye, Rayleigh-Ritz and Lanczos methods for symmetric matrix pencils (submitted).

6. T. A. Manteuffel, The Tchebychev iteration for nonsymmetric linear systems, Numer. Math. 28 (1977), 307-327.

7. C. Paige, The computation of eigenvalues and eigenvectors of very large sparse matrices, $\mathrm{Ph} . \mathrm{D}$. dissertation, University of London, 1971.

8. B. N. Parlett, The symmetric eigenvalue problem, Prentice-Hall, Englewood Cliffs, N.J., 1980.

9. B. N. Parlett, D. R. Taylor, and Z. A. Liu, A look-ahead Lanczos algorithm for unsymmetric matrices, Math. Comp. 44 (1985), 105-124. 
10. Y. Saad, On the rate of convergence of the Lanczos and block Lanczos methods, SIAM J. Numer. Anal. 17 (1980), 687-706.

11. _ Projection methods for solving large sparse eigenvalue problems, Matrix Pencils (A. Ruhe and B. Kagstrom, eds.), Lecture Notes in Math., vol. 973, Springer, New York, 1983, pp. 221-244.

12. D. S. Scott, How to make the Lanczos algorithm converge slowly, Math. Comp. 33 (1979), 239-247.

13. Q. Ye, Variational principles and numerical algorithms for symmetric matrix pencils, Ph.D. thesis, University of Calgary, 1989.

Department of Mathematics and Statistics, University of Calgary, Calgary, AlBERTA T2N 1N4, CANADA

E-mail address: ye@uncamult.bitnet 